\title{
Relationship between physician-based assessment of disease activity, quality of life, and costs of ulcerative colitis in Poland
}

\author{
Paweł Kawalec ${ }^{1}$, Ewa Stawowczyk ${ }^{2}$ \\ ${ }^{1}$ Drug Management Department, Institute of Public Health, Faculty of Health Sciences, Jagiellonian University Medical College, \\ Krakow, Poland \\ ${ }^{2}$ StatSoft Polska Sp. z o.o., Krakow, Poland
}

Gastroenterology Rev 2018; 13 (1): 61-68

DOI: https://doi.org/10.5114/pg.2018.74568

Key words: ulcerative colitis, quality of life, indirect costs, productivity loss.

Address for correspondence: Paweł Kawalec MD, PhD, Institute of Public Health, Jagiellonian University Medical College, 20 Grzegórzecka St, 31-531 Krakow, Poland, phone: +48 1242413 90, fax: +48 12421 74 47, e-mail: pawel.kawalec@uj.edu.pl

\begin{abstract}
Introduction: Ulcerative colitis (UC) is an idiopathic inflammatory bowel disorder, which requires lifelong treatment. It generates substantial direct and indirect costs, and significantly affects the quality of life, especially in the active state of the disease.

Aim: To evaluate the direct and indirect costs of UC as well as to assess disease activity and quality of life reported by patients with UC in Polish settings.

Material and methods: A questionnaire, cross-sectional study among UC patients as well as physicians involved in the therapy of the patients was conducted. The Clinical Activity Index (CAl) was used to assess disease activity, and the WPAl questionnaire to assess productivity loss. The quality of life was presented as utility calculated using the EQ-5D-3L questionnaire. Indirect costs included absenteeism, presenteeism, and informal care were assessed with the Human Capital Approach and expressed in euros $(€)$. The productivity loss among informal caregivers was valuated with the average wage in Poland. Correlations were presented using the Spearman's coefficient, and the between-group difference was assessed with Mann-Whitney U-test.

Results: One hundred and forty-seven patients participated in the study, including 95 working persons. Mean cost of absenteeism and presenteeism was $€ 1615.2$ (95\% Cl: 669.5-2561.0) and €3684.4 (95\% Cl: 2367.8-5001.1), respectively, per year per patient with a disease in remission. The mean yearly cost of productivity loss due to informal care was estimated to be $€ 256.6$ (range: 0.0-532.6). The corresponding values for patients with active disease were: $€ 8,913.3$ (95\% Cl: 6223.3-11,603.3), €4325.1 (95\% Cl: 2282.4-6367.8), and €2396.1 (95\% Cl: 402.0-4390.3). The between-group difference in total indirect costs, cost of absenteeism, and cost of informal care was statistically significant $(p<0.05)$. The average weighted monthly costs of therapy with particular drugs categories (e.g. mesalazine or biologic drugs) differed significantly between active disease or remission patients. The difference in utility values between patients with a disease in remission $(0.898 \pm 0.126)$ and patients with an active disease $(0.646 \pm 0.302)$ was statistically significant.

Conclusions: Our study revealed the social burden of UC and high dependency of direct and indirect costs as well as quality of life on the severity of UC in Poland. The statistically significant differences were identified in total direct and indirect cost, cost of absenteeism, cost of informal care, and health-related quality of life among patients with an active disease compared to patients with a disease in remission.
\end{abstract}

\section{Introduction}

Ulcerative colitis (UC) is an idiopathic inflammatory bowel disorder characterised by an inflammatory reaction involving the colonic mucosa $[1,2]$. The clinical course is unpredictable and marked by alternating periods of exacerbations and remissions, which may occur spontaneously or in response to treatment changes or intercurrent illnesses [3, 4]. The prevalence of UC in Europe ranges from 4.9 to 505 per 100,000 people, while in North America it ranges from 37.5 to 248.6 per 100,000 people [5]. People affected with UC require expensive, lifelong treatment, which generates great direct costs to the public payer. Additionally, it has a significant impact on the quality of life, especially in the active state of the disease. Ulcerative colitis is assumed to impose a considerable medical and societal burden, especially when the disease is active. 
Indirect costs or productivity losses are the labour earnings that are forgone as a result of an adverse health outcome, i.e. illness, death, side effects, or time spent on treatment. Indirect costs consist of two major components: absenteeism and presenteeism. Absenteeism refers to the number of days on sick leave, periods of unemployment caused by a disease, and early leaving of the labour market due to sickness. Presenteeism refers to a situation when a sick person is present at work but his or her own productivity is lower than average due to the disease [6]. There are two main methods to calculate the indirect costs: the Human Capital Approach, and the Friction Cost Approach. The Human Capital Approach (HCA) converts the value of work that will not be done in the future due to disease into the real costs from a social perspective. The HCA can also take into account the loss of productivity associated with early retirement or early death of the patient. The HCA is based on the assumption that work not done due to disease is a decrease of human capital and is a burden to society. The Friction Cost Approach (FCA) considers productivity losses until a new person is employed as a substitute for the sick one. The FCA is based on the assumption that society can replace a sick person in order to prevent productivity losses. This method requires access to detailed economic data and is more sophisticated than HCA, which is probably the reason why the vast majority of studies use the HCA during calculation of indirect costs [6]. Indirect costs calculated with FCA methodology are generally much lower than those obtained with HCA.

\section{Aim}

We conducted a cross-sectional study to assess the relationship between the disease activity assessed by physicians and corresponding direct and indirect costs as well as quality of life of patients with UC.

\section{Material and methods Study design}

A questionnaire-based, self-report survey was used to collect data on direct costs, health-related quality of life, and productivity loss of UC patients in Poland. Alongside this, disease activity was assessed with a survey carried out among physicians, and the general characteristics of patients with UC were collected.

The study was performed in a group of 147 patients with a diagnosis of UC as well as among their physicians in the period from mid-August to September 2016 (cut-off date: 30 September 2016); volunteers aged 18 years or older, with a diagnosis of UC, from randomly selected inpatient and outpatient medical centres in Poland, were enrolled in the study. No exclusion crite- ria that would preclude participation were used among patients who met the inclusion criteria. Centres from different regions of Poland were included to get a sample of patients with the greatest representativeness for the Polish population. The whole country was divided into five regions, and in each of these regions the random selection of medical centres engaged in UC therapy was performed to obtain a heterogeneous population of UC patients. All calculations were carried out for the general population of patients with UC and also for subgroups of patients with active disease and with disease in remission. The differences of obtained values in those two groups were also assessed.

\section{Questionnaire}

The questionnaire consisted of two parts. Part "A" was designed to be completed by the physicians and included questions that enable an assessment of disease activity but also a current pharmacological treatment. Part "B" was designed to be completed by UC patients and included questions concerning the general characteristics of patients (age, sex, place of living, date of disease onset, education, comorbidities), quality of life, direct costs, influence of the disease on usual activities, and paid and unpaid work. Both parts were designated with a matching blank patient's acronym/number assigned by physicians.

We used a clinical activity index (CAI), also known as the Rachmilewitz index, to assess the disease activity. Within this scale physicians who take care of UC patients assess the disease activity taking into account the following parameters: number of stools, blood in stools, global assessment, abdominal pain or cramps, body temperature, extraintestinal manifestations, and results of laboratory test. The CAI score ranges from 0 to 29 (higher score means more severe disease). Within this scale it is possible to categorise two types of patients: those with inactive disease/remission (CAl score $<5$ ) and those with active disease (CAl score $\geq$ 5) [7].

Health-related quality of life of the patients was assessed with the EuroQol questionnaire five-dimension, three-level version (EQ-5D-3L) [8]. In order to calculate the utility weights, the responses to the EQ-5D-3L were evaluated with the Polish tariffs [9].

To assess the productivity loss the Work Productivity and Activity Impairment (WPAI) questionnaire was used, which is a standard analytic tool commonly used to assess absenteeism and presenteeism due to a disease [10]. After consultation with experts, additional questions were added to include also the part-time employees, patients on rehabilitation benefit, or other professionally inactive patients (on illness benefit, unable 
to work, still studying, inactive due to other reasons). Questions concerning informal care were also included in the questionnaire.

\section{Resource evaluation}

The Human Capital Approach was used to estimate indirect costs due to absenteeism and presenteeism. A macroeconomic indicator for Poland was considered - gross domestic product (GDP) per working hour of a person with a professional activity in Poland in the year 2015 [11]. The correction factor of 0.65 was used, which is the conventional mean value of output elasticity of labour according to the Cobb-Douglas function of production [12]. The final unit cost of productivity loss at paid work per hour was estimated to be €8.15 (the exchange rate valid for 2016 was $€ 1=4.36$ PLN). The informal care included the time inputs of relatives without payment. The unit cost was estimated to be $€ 5.90$, which was an average income per hour of work in the third quarter of 2016 in Poland [11].

\section{Statistical analysis}

Continuous variables were summarised using means or medians and standard deviations (SDs) or 95\% confidence intervals ( $\mathrm{Cls}$ ) or ranges, while nominal variables were summarised using frequencies. Mann-Whitney $U$ test for continuous variables was used to compare the subgroups - patients with active disease and patients with disease in remission.

Based on dosage of particular drugs indicated by patients, the monthly therapy costs from public payer's perspective (reimbursement) and the patient's perspective (co-payment) were calculated for each patient.

The relationship between the disease activity, measured with CAI scale, and both the indirect costs and utility weights was assessed with Spearman's rank correlation coefficient.

Additionally, data on total indirect costs were presented on box-whisker plots, where a point presents a median value, a box presents the first and third quartiles, and whiskers present minimum and maximum values. The relationship between cost of absenteeism, total indirect cost, utility weights, and disease activity (CAl score) was presented on scatterplots. Statistical analyses were performed using Statistica ${ }^{\oplus}$.

\section{Results}

\section{General characteristics}

We obtained 147 completed questionnaires from patients fulfilling the inclusion criteria, aged from 18 to 79 years (average age: $39.0 \pm 13.4$ years; median value: 36.0 years); the total response rate was estimated at $82 \%$. The general characteristics of all patients are presented in Table I.

The majority of patients completed the questionnaire during the ambulatory visit (69.2\%), while the others completed the questionnaire during the hospitalisation, which was longer than 1 day (24.7\%) or equalled 1 day (6.2\%). Physicians assessed the extent of involvement in accordance with Montreal classification as: left-sided UC in $43.5 \%$ of patients, extensive UC in $30.3 \%$, and ulcerative proctitis in $26.2 \%$. The average value of CAl was 4.9 (95\% Cl: 3.9-5.8, median value: 3.0). The severity of the disease was also assessed by physicians. Disease in clinical remission was seen in $27.4 \%$ of patients, $36.8 \%$ had mild disease, $21.1 \%$ had moderate disease, and $14.7 \%$ of patients had severe disease.

The average age at diagnosis was $31.7 \pm 12.8$ years (median value: 29.0 years). The general wellbeing was moderate and equalled 6.3 (95\% Cl: 5.9-6.7, median

Table I. General characteristics of all and working patients with UC included in the study

\begin{tabular}{|c|c|c|c|}
\hline \multicolumn{2}{|l|}{ Characteristic } & All patients & Working patients \\
\hline \multicolumn{2}{|l|}{ Age [years] } & $39.03 \pm 13.42$, range: $18-79$ & $38.83 \pm 10.27$, range: $22-68$ \\
\hline \multicolumn{2}{|l|}{ Male } & $69(46.94 \%)$ & $44(46.32 \%)$ \\
\hline \multicolumn{2}{|c|}{ Disease onset [years] } & $31.65 \pm 12.76$, range: $3-70$ & $31.66 \pm 10.72$, range: $3-63$ \\
\hline \multirow[t]{3}{*}{ Place of living } & City $\geq 100,000$ citizens & $95(66.43 \%)$ & $66(72.53 \%)$ \\
\hline & City $<100,000$ citizens & $39(27.27 \%)$ & $22(24.18 \%)$ \\
\hline & Village & $9(6.29 \%)$ & $3(3.30 \%)$ \\
\hline \multirow[t]{3}{*}{ Education } & Higher & $55(38.19 \%)$ & $43(46.24 \%)$ \\
\hline & Secondary & $80(55.56 \%)$ & $49(52.69 \%)$ \\
\hline & Basic & $9(6.25 \%)$ & $1(1.08 \%)$ \\
\hline \multicolumn{2}{|l|}{ Comorbidities } & 37 (25.17\%) & $23(24.21 \%)$ \\
\hline
\end{tabular}




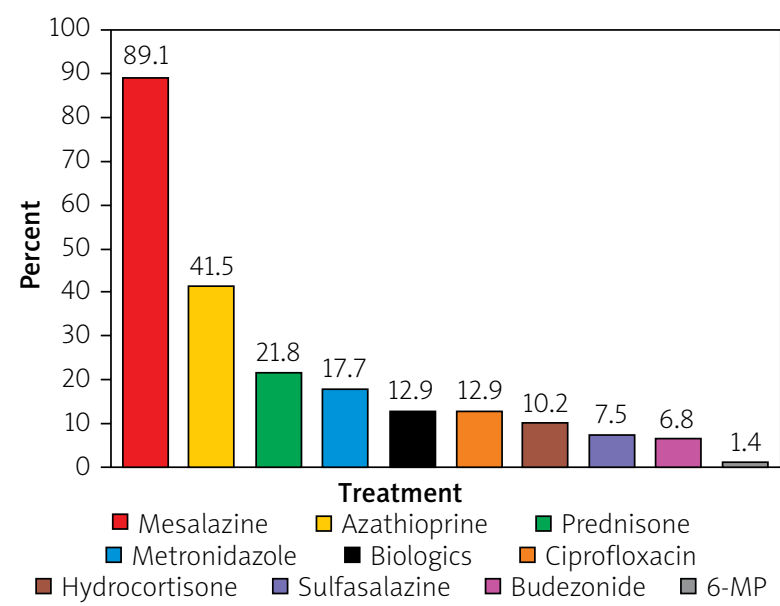

Figure 1. Pharmacological treatment of UC

value: 6.0), while the minimum and maximum possible values were: 1 (the worst) and 10 (the best), respectively. The influence of the disease on daily activities was rather limited and equalled 3.8 (95\% Cl: 3.2-4.3, median value: 3.0 ), while the minimum and maximum possible values were: 1 (no influence) and 10 (the highest influence), respectively.

Data on employment status was collected for all 147 patients, of which 95 (64.6\%) were currently working.

The general characteristics of working patients were presented in Table I. Among all 95 working patients, 89 had paid job, one was retired, and one was receiving rehabilitation benefit. One patient was still studying and also working, but the form of employment was not mentioned. Three patients did not determine the form of employment.

For all working patients, data on disease activity with the CAI questionnaire were available. The average value of CAI was 5.1 (95\% Cl: 3.9-6.3; median value: 3.0). Among 95 working patients with UC, $64.2 \%$ had remission according to CAI score.

\section{Direct costs}

Patients reported on average $1.6 \pm 1.3$ (range: 0.0-8.0, median value: 1.0$)$ medical consultations per month, from which on average 0.8 were private (range: 0.0-4.0, 95\% Cl: 0.7-1.0; median value: 1.0$)$. The cost of one private consultation was on average $€ 30.6 \pm 6.7$ (range: 18.3-45.9, median value: 29.8).

Patients reported on average 0.6 hospitalisations per month (range: $0.0-6.0,95 \% \mathrm{Cl}: 0.4-0.8$ ) and the median length of stay was 3.0 days (range: 0.0-21.0, average value: $6.1,95 \% \mathrm{Cl}: 4.3-7.8)$. Hospitalisations are fully reimbursed from public funds (by National Health Fund) in Poland, and its cost ranged from about $€ 572$ to $€ 954$.
The majority (48.0\%) of patients spent from $€ 23$ to $€ 46$ monthly on drugs used in the treatment of UC, and only $1.4 \%$ spent $€ 92-€ 138$. The additional expenditures such as informational materials, dietary supplements are lower $-42.5 \%$ of patients spent from $€ 0$ to $€ 23$ monthly.

Almost $90 \%$ of patients took mesalazine, while biological treatment of UC (adalimumab or infliximab) was used in almost $13 \%$ of patients (Figure 1 ). The average weighted monthly costs of therapy with particular drugs per patient differed significantly in between both considered groups: patients with active disease and patients with disease in remission (Table II).

\section{Absenteeism and presenteeism \\ All working patients}

The average annual number of days off work due to the illness among working patients was 66.3 (95\% Cl: 45.8-86.8, range: 0-258). The average reduction of work productivity due to absenteeism was $25.7 \%$ (95\% Cl: 17.8-33.7\%, range: 0-100\%). Mean cost of absenteeism was €4161.1 (95\% Cl: 2839.1-5483.0) per year per working patient.

Average productivity loss at paid work of a working patient was 37.0\% (95\% Cl: 29.6-44.4; range: 0-100\%), which represents the extent of presenteeism. Mean cost of presenteeism was €3907.9 (95\% Cl: 2815.8-5000.0) per year per patient with any occupational activity.

Total average reduction of productivity due to UC (reduction resulted from both absenteeism and presenteeism) was $45.1 \%$ (95\% Cl: 36.9-53.3\%, range: 0-100\%). Total average indirect cost per year per working patient was $€ 8069.0$ (95\% Cl: 6532.9-9605.1).

The cost of absenteeism and total indirect cost was moderately-to-strongly correlated with CAI score. The Spearman's coefficients were 0.6475 and 0.5459 , respectively (Figures 2, 3). No statistically significant correlation was found for cost of presenteeism and CAI score.

\section{Patients with a disease in remission}

The average annual number of days off work due to illness among working patients with a disease in remission was 27.4 (95\% Cl: 10.8-44.0, range: 0-258). The average productivity loss due to absenteeism was $11.1 \%$ (95\% Cl: 4.4-17.9\%, range: 0-100\%). Mean cost of absenteeism was $€ 1615.2$ (95\% Cl: 669.5-2561.0) per year per patient with any occupational activity.

Average productivity loss of paid work for a working patient with a disease in remission was $24.3 \%(95 \% \mathrm{Cl}$ : $16.1-32.4 \%$, range: $0-100 \%)$. Mean cost of presenteeism was $€ 3684.4$ (95\% Cl: 2367.8-5001.1) per year per patient with any occupational activity.

Total average reduction due to UC (reduction resulted from both absenteeism and presenteeism) was 
Table II. Monthly pharmacotherapy costs per patient

\begin{tabular}{|c|c|c|c|c|}
\hline \multirow[t]{2}{*}{ Drugs } & \multirow{2}{*}{$\begin{array}{c}\text { Perspective of public } \\
\text { payer (reimbursement) } \\
\text { and patient (co-payment) }\end{array}$} & \multicolumn{2}{|c|}{ Monthly costs $[€]$} & \multirow[t]{2}{*}{$P$-value } \\
\hline & & Active disease & Remission & \\
\hline \multirow[t]{2}{*}{ Biologics } & Public payer & 236.38 & 52.40 & 0.0005 \\
\hline & Patient & 0.00 & 0.00 & - \\
\hline \multirow[t]{2}{*}{ Prednisone } & Public payer & 4.29 & 1.02 & $<0.0005$ \\
\hline & Patient & 1.04 & 0.25 & $<0.0005$ \\
\hline \multirow[t]{2}{*}{ Hydrocortisone } & Public payer & 10.63 & 0.78 & $<0.0005$ \\
\hline & Patient & 4.56 & 0.33 & $<0.0005$ \\
\hline \multirow[t]{2}{*}{ Sulfasalazine } & Public payer & 1.19 & 1.76 & 0.5621 \\
\hline & Patient & 0.13 & 0.20 & 0.5621 \\
\hline \multirow[t]{2}{*}{ Mesalazine } & Public payer & 28.90 & 23.91 & 0.0247 \\
\hline & Patient & 15.70 & 12.99 & 0.0247 \\
\hline \multirow[t]{2}{*}{ Azathioprine } & Public payer & 4.11 & 2.80 & 0.0616 \\
\hline & Patient & 0.70 & 0.43 & 0.0235 \\
\hline \multirow[t]{2}{*}{ Mercaptopurine } & Public payer & 0.40 & 0.00 & 0.0501 \\
\hline & Patient & 0.05 & 0.00 & 0.0501 \\
\hline \multirow[t]{2}{*}{ Budesonide } & Public payer & 1.94 & 8.98 & 0.0981 \\
\hline & Patient & 0.01 & 0.07 & 0.0981 \\
\hline \multirow[t]{2}{*}{ Ciprofloxacin } & Public payer & 1.21 & 0.08 & $<0.0005$ \\
\hline & Patient & 2.68 & 0.19 & $<0.0005$ \\
\hline \multirow[t]{2}{*}{ Metronidazole } & Public payer & 0.00 & 0.00 & - \\
\hline & Patient & 13.25 & 2.49 & $<0.0005$ \\
\hline
\end{tabular}

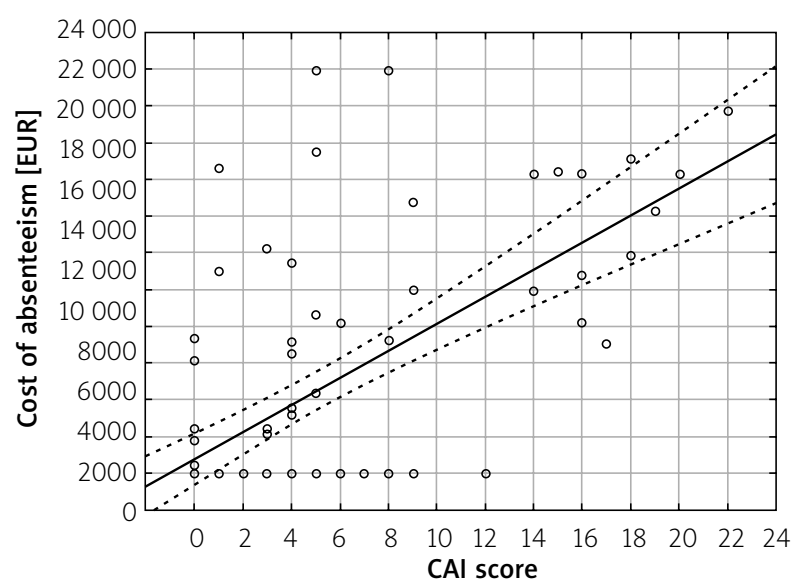

Figure 2. The relationship between cost of absenteeism and CAl score

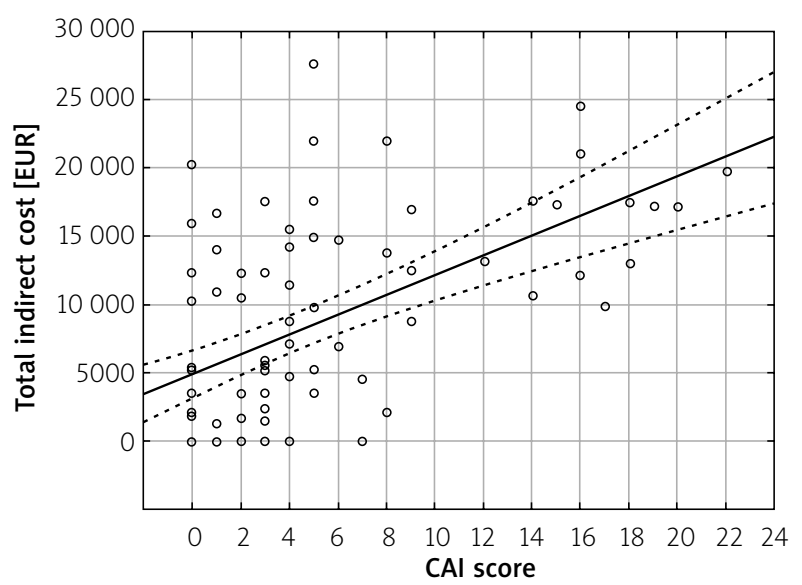

Figure 3. The relationship between total indirect cost and CAl score 
$30.2 \%$ (95\% Cl: 21.2-39.3\%, range: 0-100\%). Total average indirect cost per year per working patient was $€ 5299.7$ (95\% Cl: 3719.6-6879.8).

\section{Patients with an active disease}

The average annual number of days off work due to the illness among working patients with an active disease was 138.8 (95\% Cl: 99.4-178.3, range: 0-258). The average productivity loss due to absenteeism was 52.9\% (95\% Cl: 37.7-68.1\%, range: 0-100\%). Mean cost of absenteeism was €8913.3 (95\% Cl: 6223.3-11,603.3) per year per patient with any occupational activity.

Average productivity loss at paid work of working patient with active disease was $60.6 \%(95 \% \mathrm{Cl}: 49.5-$ $71.7 \%$, range: $0-100 \%)$. Mean cost of presenteeism was $€ 4325.1$ (95\% Cl: 2282.4-6367.8) per year per patient with any occupational activity.

Total average reduction due to UC (reduction resulted from both absenteeism and presenteeism) was 72.6\% (95\% Cl: 61.0-84.3\%, range: 0-100\%). Total average indirect cost per year per working patient was $€ 13,238.4$ (95\% Cl: 10,819.2-15,657.5).

Mann-Whitney $U$ test indicated the significant difference $(p<0.05)$ in average value of cost of absenteeism and total indirect costs between the two analysed groups: active disease and disease in remission. Median value, as well as range and $1^{\text {st }}$ and $3^{\text {rd }}$ quartiles of total indirect cost are presented in box-whiskers plot (Figure 4).

\section{Informal care}

Twenty working patients (21.3\%) declared that they require assistance in performing usual activities, which is supported by their relatives. Patients received the assistance in performing usual activities for an average

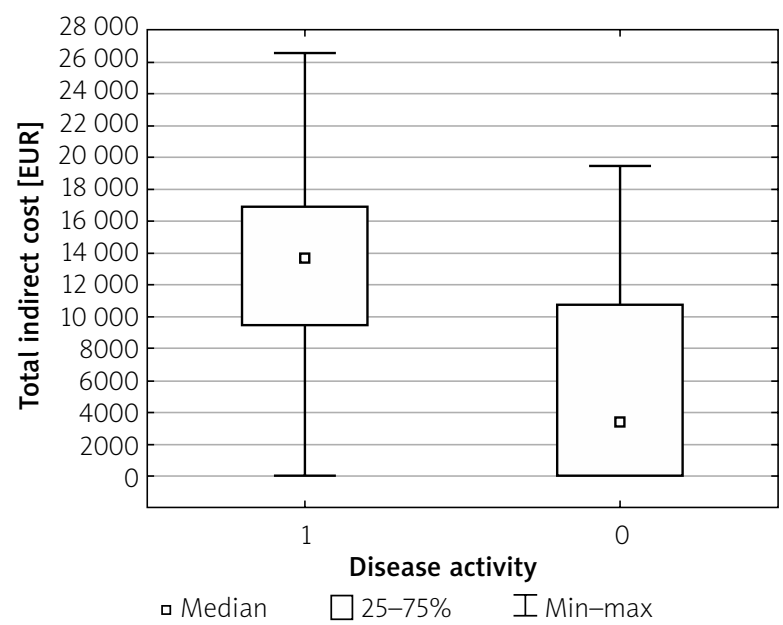

Figure 4. Total indirect costs according to disease activity: 1 - patients with active disease, 0 - patients with disease in remission of 16.6 (95\% Cl: 6.0-27.2, median value was 9.0) hours per week. The mean cost of productivity loss due to informal care was estimated to be $€ 1000.8$ (95\% Cl: 277.1-1724.5) per year.

Similar analyses were performed for two subgroups: working patients with active disease and working patients with disease in remission.

In the first group 39.4\% patients declared that they require assistance in performing usual activities, while among working patients with a disease in remission only $11.5 \%$ declared the need of such support. Patients with an active disease and with a disease in remission received assistance in performing usual activities for an average of 20.8 (95\% Cl: 5.0-36.5, median value: 12.0 ) and 8.3 (range: 4.0-25.0, median value: 5.0 ) h/week, respectively. The mean yearly cost of productivity loss due to informal care was estimated to be $€ 2396.1$ (95\% Cl: 402.0-4390.3) among working patients with active disease and $€ 256.6$ (range: 0.0-532.6) among working patients with disease in remission.

Mann-Whitney $U$ test indicated the significant difference $(p<0.05)$ in the average cost of informal care between two analysed groups: patients with active disease and with disease in remission.

\section{Health-related quality of life}

Data on the quality of life were reported by 144 (97.1\%) respondents. The average utility index in all patients was estimated to be $0.811 \pm 0.237$, and 0.809 \pm 0.234 for working patients. Patients with active disease had significantly lower utility indexes $(0.646$ \pm 0.302 ) compared to patients with a disease in remission $(0.898 \pm 0.126)$. The corresponding values for working patients with active disease and with disease in remission were $0.665 \pm 0.259$ and $0.889 \pm 0.174$, respectively. The difference in utility values between the above groups was statistically significant based on Mann-Whitney $U$ test $(p<0.05)$.

Utility indexes among study participants were correlated with CAI score (Spearman's coefficient of -0.702 for all patients, Figure 5, and -0.655 for working patients, $p<0.05$ )

The relationship between the utility indexes and the indirect costs was assessed in working patients. The correlation between the above parameters was negative and moderate and equalled -0.445 , which means that higher indirect costs were observed among patients with lower utility weights.

\section{Discussion}

Within this study we assessed the disease activity with CAI questionnaire as well as costs and quality of life of patients with UC in Poland. The study was carried 
out among diagnosed adult patients with UC and their physicians, who assessed the diseases activity and severity, and current medical treatment. Additionally, we assessed the relationship between the disease activity and indirect costs as well as utility weights. It was proven that the activity of the disease significantly affected total indirect costs and indirect costs due to absenteeism. Presenteeism - the productivity of sick person at work - was not dependent from disease activity. The reason for this was probably the fact that patient who felt bad, stayed at home and took a day (days) off work due to the illness, which influenced absenteeism and did not affect presenteeism. Conducted analysis included also cost of informal care, which was calculated based on the need of assistance in performing usual activities and the number of hours per week of such help. The above cost was also dependent from the disease activity, i.e. it was significantly higher for patients with active disease compared with patients with disease in remission. We calculated also direct costs, mainly resulting from pharmacotherapy. All drugs that were indicated by the physicians were included in the analysis, and the treatment costs with each drug for each patient were calculated. Finally, it was shown that the pharmacotherapy costs of almost all drugs differed significantly comparing patients with active or remission stage of disease. This resulted from the fact that patients in remission much less frequently need pharmacotherapy.

There have been no previous studies that have assessed the disease activity measured with CAl questionnaire, direct and indirect costs, and quality of life of Polish patients with UC. Therefore, it seemed necessary to conduct such a study. Indirect costs included both absenteeism and presenteeism, and also the cost of informal care, which is very rarely assessed in cost studies. The main calculations were made for all patients and also for subgroups: patients with active disease and with disease in remission.

In the calculations of indirect costs only working patients with diagnosed UC were included, who constituted about $65 \%$ of all study participants.

The main advantage of this study was the size of the study population and its diversification. The study was carried out in randomly selected, Polish medical centres from the following cities: Poznan, Wroclaw, Opole, Katowice, Lodz, and Warsaw (two medical centres). The randomisation was performed to obtain a sample of patients that best represented the Polish population. The whole country was divided into five regions, and in each of those regions the random selection of medical centres engaged in UC therapy was performed. Disease activity was assessed with a reliable questionnaire - CAl - by physicians, which is an additional strength of this

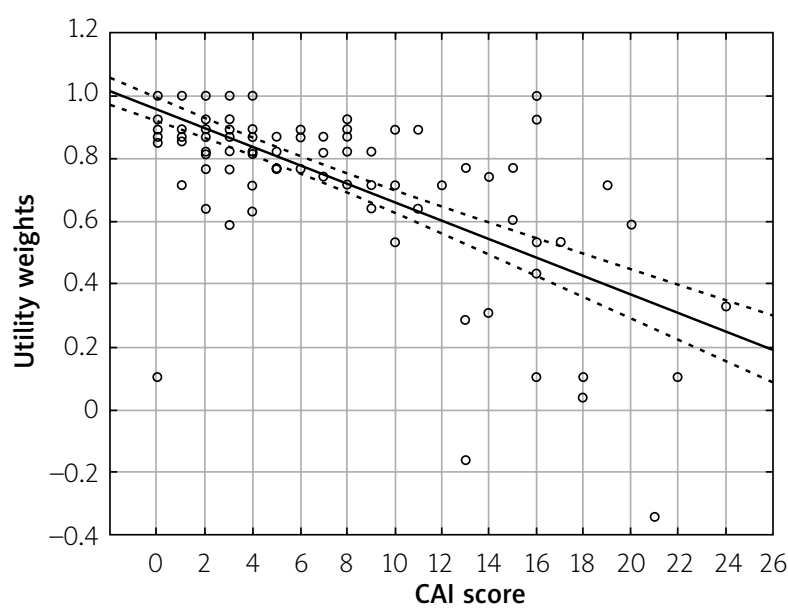

Figure 5. The relationship between utility weights and CAl score

study because patient-based assessment of disease activity may be less precise. Answers were collected during clinical examination, which assured the highest reliability - the biases resulting from self-reporting were eliminated. Direct costs were assessed by the patients and did not include all possible categories, which can constitute a limitation.

Evidence on indirect costs of UC is available but typically provides information on the loss of productivity due to absence from work and due to lower productivity at work, and our study was the first one providing additional information on indirect costs as well as on the costs of informal care in Polish settings. There is lack of such information also for countries from the CEE region, which made our research quite interesting.

In order to compare our results with findings of other investigators, we performed a review of medical databases. We discovered some studies considering the association between the disease activity and quality of life or indirect costs across Europe, but such studies in Polish settings were not identified.

The study by Casellas et al. [13], which included UC patients, showed that Spearman's correlation coefficients between health-related quality of life measured with Inflammatory Bowel Disease Questionnaire (IBDQ) and clinical and colonoscopic indices were statistically significant $(p<0.01)$ and equalled from -0.67 to -0.61 and from -0.70 to -0.67 , respectively, depending on the questionnaire - full form IBDQ-36 or short form IBDQ-9. The results obtained in our analysis showed a slightly lower correlation of -0.55 , but the quality of life was measured in a different way - using an EQ-5D questionnaire, as well as disease activity, which was measured with P-CAl questionnaire. Gibson et al. [14] calculated the mean (standard deviation SD) EQ-5D-5L scores among UC patients, which were 
greater for patients in remission $(0.81(0.18))$ than for patients with active disease (0.72 (0.19), $p<0.001)$. The same dependence was observed in our analysis. Patients in remission and with mild disease had much less impairment than patients with moderate/severe disease for every type of work and activity impairment (i.e. work time missed, overall work impairment, impairment while working, activity impairment). In a study by Mandel et al. [15], among employed IBD (UC and (D) patients, absenteeism and presenteeism was reported in $25.9 \%$ and $60.3 \%$ of patients, respectively, leading to a $28 \%$ loss of work productivity and a $32 \%$ activity loss. It was significantly different according to IBD activity assessed with partial Mayo score in the case of UC. The significant difference in productivity loss between patients with active disease and patients with disease in remission was also observed in our analysis. Among 226 UC patients, moderate correlation was also observed by Taleban et al. [16] between the Mayo endoscopic score and health-related quality of life measured with the Short Inflammatory Bowel Disease Questionnaire (SIBDQ; $r=-0.56$ ), and disease activity measured with Simple Clinical Colitis Activity Index (SCCAl; $r=0.55$ ).

Our study was the first one to assess the relationship between the disease activity measured with CAI with quality of life measured with EQ-5D and indirect costs, but similar assessments were made taking into account different measures of health-related quality of life and activity of the disease. All of the identified studies confirm the results of our analysis. Health-related quality of life and productivity loss are significantly different among patients with active disease and patients with disease in remission. No studies were identified that included informal care and the influence of the disease on the daily activities, other than paid work.

\section{Acknowledgments}

The study was funded and performed according to a scientific grant (Jagiellonian University Medical College; statutory project Drug Management Department, Institute of Public Health JU MC; K/ZDS/006111).

\section{Conflict of interest}

Authors declare no conflict of interest.

\section{References}

1. Baumgart DC, Sandborn WJ. Inflammatory bowel disease: clinical aspects and established and evolving therapies. Lancet 2007; 369: 1641-57.

2. Feagan BG, Reinisch W, Rutgeerts P, et al. The effects of infliximab therapy on health-related quality of life in ulcerative colitis patients. Am J Gastroenterol 2007; 102: 794-802.
3. Kornbluth A, Sachar DB, The Practice Parameters Committee of the American College of Gastroenterology. Ulcerative Colitis Practice Guidelines in Adults: American College of Gastroenterology, Practice Parameters Committee. Am J Gastroenterol 2010; 105: 501-23.

4. Ordás I, Eckmann L, Talamini M, et al. Ulcerative colitis. Lancet 2012; 380: 1606-19.

5. Tontini GE, Vecchi M, Pastorelli L, et al. Differential diagnosis in inflammatory bowel disease colitis: state of the art and future perspectives. World J Gastroenterol 2015; 21: 21-46.

6. Krol M, Brouwer W. How to estimate productivity costs in economic evaluations. Pharmacoeconomics 2014; 32: 335-44.

7. Rachmilewitz D. Coated mesalazine versus sulphasalazine in the treatment of active ulcerative colitis. Br Med J 1989; 298: 82-6.

8. Krol M, Brouwer W. How to estimate productivity costs in economic evaluations. Pharmacoeconomics 2014; 32: 335-44.

9. Golicki D, Jakubczyk M, Niewada M, et al. Valuation of EQ-5D health states in Poland: first TTO based social value set in Central and Eastern Europe. Value Health 2010; 13: 289-97.

10. Reilly MC, Zbrozek AS, Dukes EM. The validity and reproducibility of a work productivity and activity impairment instrument. Pharmacoeconomics 1993; 4: 353-65.

11. Central Statistical Office of Poland, http://stat.gov.pl/en/ Last Access: January 2016.

12. Havik K, Mc Morrow K, Orlandi F, et al. The production function methodology for calculating potential growth rates and output gaps. Economic Papers 535, November 2014, http://ec.europa. eu/economy_finance/publications/economic_paper/2014/pdf/ ecp535_en.pdf.

13. Casellas F, Alcalá MJ, Prieto L, et al. Assessment of the influence of disease activity on the quality of life of patients with inflammatory bowel disease using a short questionnaire. Am J Gastroenterol 2004; 99: 457-61.

14. Gibson PR, Vaizey C, Black CM, et al. Relationship between disease severity and quality of life and assessment of health care utilization and cost for ulcerative colitis in Australia: a cross-sectional, observational study. J Crohns Colitis 2014; 8: 598-606.

15. Mandel MD, Bálint A, Lovász BD, et al. Work disability and productivity loss in patients with inflammatory bowel diseases in Hungary in the era of biologics. Eur J Health Econ 2014; 15 Suppl 1: S121-8.

16. Taleban S, Stewart KO, Li DK, et al. Clinical activity and quality of life indices are valid across ulcerative colitis but not Crohn's disease phenotypes. Dig Dis Sci 2016; 61: 2627-35.

Received: 14.01 .2017

Accepted: 24.05.2017 